\title{
Effect of fatty imidazolines on properties of laundry detergent compositions based on cationic surfactants
}

\author{
Divya Tripathy ${ }^{1}$ and V. K. Tyagi ${ }^{2}$ \\ ${ }^{l}$ Department of Aplied Science and Humanities, ITS Engineering College, Knowledge Park III, Greater Noida \\ INDIA \\ ${ }^{2}$ Department of Oil and Paint Technology, Harcourt Butler Technological Institute, Kanpur- 208002 INDIA
}

\begin{abstract}
The present study emphasizes on the synthesis of cationic imidazolinium surfactants based on easily available and cheap fatty materials. Surface active properties of $1 \%$ aqueous solution of imidazolinium surfactants were evaluated in terms of surface tension, interfacial tension, cmc, dispersability and emulsion stability. To evaluate the performance properties, laundry detergent compositions were made using different percentages of synthesized imidazolinium surfactants with nonionic triton $x$-100 and properties were evaluated in terms of foaming, softening, rewettability and detergency. The presence of cationic imidazolinium surfactants in laundry compositions, increased the detergency, rewettability, foam stability and softening of compositions Keywords: Imidazolinium surfactants, Surface active properties, Performance properties, Laundry detergent compositions, Nonionic surfactants
\end{abstract}

\section{Introduction}

Imidazoline surfactants belong to the category of cationic surfactants which are presently gaining importance in global detergent market due to their wide range of applications (1). These are extensively used mainly as fabric softeners and antistatic agents. Substituted imidazoline compounds possess desirable storage stability, viscosity, dispersability and fabric conditioning properties and are useful in laundry applications and various industrial applications.

Cationic imidazolines are the ammonium mono quaternary salts of $\mathrm{RnN}^{+} \mathrm{X}^{-}$type that have an asymmetrical structure(according to the tetrahedral carbon scheme) determined by the cation. They are optically active in aqueous solution in which they are ionized. Substitution of imidazolines takes place in the 1-,2-\& 3positions with a fatty acid aminoethyl chain at the first position of ring, a long chain alkyl substituent at the second position and a methyl group at the third position(3) as depicted in Fig. 1

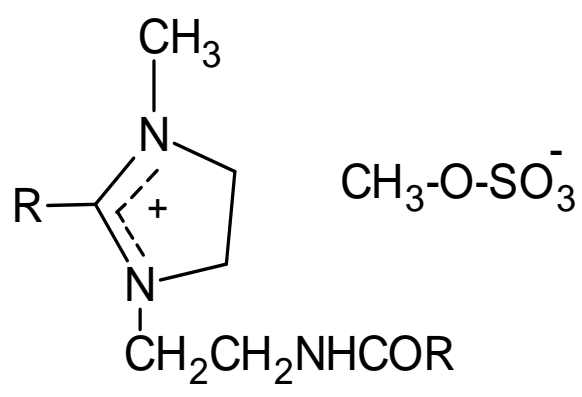

$\mathrm{R}=\mathrm{C}_{12} / \mathrm{C}_{18}$ fatty alkyl chain

Fig. 1 Structure of imidazolines

Chemically imidazoline compounds belong to the heterocyclic compounds containing five membered ring having two nitrogen atoms in the ring. Imidazolinium surfactant comprises of a five membered positively charged hydrophobic head group and a hydrobhobic chain which is generally a fatty alkyl group obtained from $\mathrm{C}_{12}-\mathrm{C}_{18}$ saturated/unsaturated fatty acids/alcohols. These different hydrobhobic chains are responsible for the different surface active and performance properties of imidazolinium surfactants and also make them suitable for different industrial applications.

The most widely used representative of this group is the tallow alkyl derivative. It is applied as a liquid and is easily processed. Other approximate composition of the fatty alkyl groups are given in Table1 
Table 1 Approximate composition of the fatty alkyl groups

\begin{tabular}{|lcccc|}
\hline & $\begin{array}{c}\mathbf{C}_{\mathbf{1 4}} \\
\text { Myristyl }\end{array}$ & $\begin{array}{c}\mathbf{C}_{16} \\
\text { Palmityl }\end{array}$ & $\begin{array}{c}\mathbf{C}_{18} \\
\text { Stearyl }\end{array}$ & $\begin{array}{c}\mathbf{C}_{18} \\
\text { Oleyl }\end{array}$ \\
\hline Oleyl & - & 5 & 20 & 75 \\
Tallow & 5 & 30 & 20 & 45 \\
Hydrogenated tallow & 5 & 30 & 65 & - \\
Stearyl & - & 5 & 95 & - \\
\hline
\end{tabular}

The unsaturated $\mathrm{C}_{18}$ contains one double bond in a cis isomer configuration. "Unsaturation" in a fat tends to lower the melting point and make it a liquid at room temperature. The saturated $\mathrm{C}_{18}$ contains no double bonds, giving rigidity to the molecule, raising the melting point and reducing fluidity.

The most widely used representative of this group is the tallow alkyl derivative (2). It is supplied as a liquid and is easily processed in fabric softeners. The imidazolinium derivative with a hydrogenated tallow chain gives better softening effect than the nonhydrogenated formulation to obtain a finished product. The oleyl derivative has recently gained importance because it has less hydrophobizing effect with relatively good softening performance. Thus textile treated with it keeps almost all their natural absorbency properties. In practice, imidazolinium compounds are frequently blended with distearyl dimethyl ammonium compounds to attain a higher degree of softening performance.

In the present study surface active properties and laundry performance properties of imidazolinium surfactants having C12-C18 saturated and unsaturated fatty alkyl chain

have been determined.

\section{Materials}

Chemicals used for the synthesis of imidazolines and their quaternized salts were of analytical grade obtained from M/s Qualigens and E. Merck, Mumbai.

\subsection{Preparation of Imidazolines}

\section{Experimental}

In an open Pyrex vessel $(500 \mathrm{ml}) 20 \mathrm{mmol}$ of diethylenetriamine, $40 \mathrm{mmol}$ of the corresponding fatty acid (stearic, palmitic, myristic, lauric, oleic and palm fatty acids) and $20 \mathrm{~g}$ of calcium oxide were carefully mixed. The reaction mixture was irradiated using power $(850 \mathrm{~W})$ of microwave oven for required time (as given in Table 2) and final temperature was noted down. The reaction mixture was allowed to reach at room temperature. Ethyl acetate was added $(80 \mathrm{ml})$ and the mixture was heated until boiling and filtered off while hot, and the filtrate was concentrated under vacuum to dryness, yielding the corresponding product as a white to yellowish brown, solid to semisolid substances.

\subsection{Preparation of quaternary imidazolinium salts}

Imidazoline ( $3.6 \mathrm{~g}$ ) was taken in $120 \mathrm{~g}$ of isopropanol and $1.5 \mathrm{~mol}$ of dimethyl sulfate was added to the solution and the reaction mixture was refluxed at $80^{\circ} \mathrm{C}$ with constant stirring for 4 hours, which was then cooled to room temperature and dried to recover imidazolinium salts.

\subsection{Instrumental analysis}

FT-IR, ${ }^{1} \mathrm{H}$ NMR and ${ }^{13} \mathrm{C}$ - NMR spectra of synthesized surfactants were carried out.

FT-IR spectra were obtained by using model vector 22 , Germany. $\mathrm{KBr}$ pellets of the sample were subjected to analysis of 20 scans.

${ }^{1}$ H NMR spectra were carried out by FT NMR system of A Lambda JEOL JNM-LA 400. Chemical shifts are reported as (ppm) relative to tetramethyl-silane.

\subsection{Methods used for evaluation surface active properties of imidazolinium quats}

\subsubsection{Determination of solubility of quaternary imidazolinium salts}

- Solubility of imidazolinium salts were checked by dissolving them in the solvents of different polarity.

- Imidazolinium salts of palm fatty acids, oleic acid and $\mathrm{C}_{18}$ saturated fatty acid were less soluble in water and $10 \%$ alcohol $\mathrm{v} / \mathrm{v}$ was added to make their aqueous solutions.

\subsubsection{Determination of melting point}

Melting point can be conveniently determined by the melting point apparatus. A small quantity of compound was finely powdered between the pieces of filter papers and introduced in a glass capillary tube 
closed at one end. The temperature at which the compounds melt and becomes almost transparent was noted. This is the melting point of that compound.

\section{4. 3 Interfacial tension}

Interfacial tension was measured with a DCAT tensiometer using $0.1 \%$ wt. solution .The measurement of the surface tension was done with a Wilhemy plate. The measurement of interfacial tension was done in three steps. First the buoyancy of the probe in the liquid with the lower density (oil) was measured. In the second step the surface of the liquid with higher density (surfactant solution) was detected. The third step was the real measurement of interfacial tensions between the two not miscible liquids.

\subsubsection{Determination of surface tension and CMC (Critical Micelle Concentration)}

Surface tension and CMC of surfactants solutions were determined at $25{ }^{\circ} \mathrm{C}$ with the help of DCAT tensiometer by using $0.1 \%$ wt. solution. The measurement of the surface tension was done with a Wilhemy plate, made of platinum-iridium (3).

\subsubsection{Determination of cationic content of imidazolinium quat}

Cationic matter of imidazolinium quats were determined as per BIS: 4955, 1978; p-8.

Apparatus:

(i) Volumetric flasks: 1000,500 and $250 \mathrm{ml}$.

(ii) Stoppered graduated cylinder $-100 \mathrm{ml}$

(iii) Burette: $25 \mathrm{ml}$

(iv) Pipette: $10 \mathrm{ml}$

Reagents:

(i) Chloroform

(ii) Standard sodium lauryl sulphate solution: $(0.004 \mathrm{M})$ Weigh to the nearest $1 \mathrm{mg}$, between 1.14 and $1.16 \mathrm{~g}$ of sodium lauryl sulphate and dissolve in $200 \mathrm{ml}$ of water. Transfer to a ground-glass stoppered 1 litre onemark volumetric flask and dilute to the mark with water.

(iii) Standard benzethonium chloride solution (Hyamine solution): (0.004M). Dry the benzethonium chloride at $105^{\circ} \mathrm{C}$, weigh $1.792 \mathrm{~g}$ to the nearest $1 \mathrm{mg}$, dissolve in water, dilute to 1 litre.

(iv) Methylene blue solution (0.005\%): Dissolve $0.05 \mathrm{~g}$ of methylene blue, $50 \mathrm{~g}$ of sodium sulphate and $6.8 \mathrm{ml}$ of concentrated sulphuric acid in water and make up the volume to 1 litre with water.

(v) Imidazolinium quat solution $(0.004 \mathrm{M})$ : Weigh adequate quantity of imidazolinium quat and dissolve in 20 $\mathrm{ml}$ of distilled ethanol and then make up $250 \mathrm{ml}$ with distilled water.

\section{Procedure :}

Standardization of sodium lauryl sulphate solution: Pipette out the $10 \mathrm{ml}$ of standard benzethonium chloride solution in a $100 \mathrm{ml}$ graduated cylinder provided with a glass stopper then added $15 \mathrm{ml}$ of chloroform and $25 \mathrm{ml}$ of methylene blue solution to the cylinder and shaked well. The lower chloroform layer coloured to blue colour. Add from the burette sodium lauryl sulphate solution slowly; initially in portions of $0.2 \mathrm{ml}$. After each addition, stoppered the cylinder, shaked well and allowed the phase to separate. Initially the chloroform phase will be coloured blue. Towards the end the colour would start migrating to the aqueous layer. Noted down the reading at which the colour intensity in both the phases is the same when viewed under standard conditions of light.

\section{Calculation:}

$$
\text { Molarity of } S L S\left(T_{2}\right)=\frac{10 T_{1}}{V_{1}}
$$

where,

$\mathrm{T}_{1}=\quad$ Molarity of hyamine solution (i.e. $0.004 \mathrm{M}$ )

$\mathrm{V}_{1}=\quad$ Volume of SLS solution added

Determination of cationic content in imidazoline quat: Pipette $10 \mathrm{ml}$ of standard sodium lauryl sulphate solution in a $100 \mathrm{ml}$ graduated cylinder provided with a glass stopper, Add $15 \mathrm{ml}$ of chloroform \& $25 \mathrm{ml}$ methylene blue solution to the cylinder. Shake well the contents.

Add from burette alcohol solution of imidazolinium quat slowly, initially in portions of $0.2 \mathrm{ml}$. Proceed as described in std. of SLS solution. 
Calculation:

Calculate the cationic matter as follows

Molarity of esterquat $\left(T_{3}\right)=\frac{10 T_{2}}{V_{2}}$

where,

$\mathrm{T}_{2}=$ Molarity of SLS solution.

$\mathrm{V}_{2}=$ Volume of imidazolinium quat solution.

Cationic matter percent by mass $=\frac{T_{3} \times \text { Molecular wt. of imidazolinium quat } \times 100}{w \text { t.of sample present in } 250 \mathrm{ml} \text { of solution } \times 4}$

\subsubsection{Determination of dispersing power}

To measure the dispersing power, $\mathrm{TiO}_{2}$ was added separately to $1 \%$ of surfactant solutions and the mixture were shaken and allowed to stand (4). Evaluations were carried out by looking at the height and cloudiness of each solutions and noting the scores.

\subsubsection{Determination of emulsion stability}

Emulsion stability determination was done by preparing $10 \mathrm{ml}$ of $20 \mathrm{mmol}$ aqueous solution of surfactants and $5 \mathrm{ml}$ of toluene at $40{ }^{\circ} \mathrm{C}$. The emulsion stability was determined as the time of separation of water $(9 \mathrm{ml})$ from the emulsion layer (5).

\subsection{Methods used for performance evaluation of quaternary imidazoline surfactants}

To determine the performance properties of synthesized imidazolinium surfactants with nonionic surfactants, surfactant compositions were made using four different percentages of $1 \%$ of each cationic imidazolinium surfactant solutions with $1 \%$ aqueous solution of nonionic surfactant (Triton Nonionic X-100) that has come to be recognized as the performance standard. The designed compositions were as follows:

\begin{tabular}{|c|c|c|c|c|c|}
\hline $\begin{array}{l}\text { S1= } \\
S: N(05: 95)\end{array}$ & $\begin{array}{l}\mathrm{P} 1= \\
\mathrm{P}: \mathrm{N}(05: 95)\end{array}$ & $\begin{array}{l}M 1= \\
M: N(05: 95)\end{array}$ & $\begin{array}{l}\mathrm{L} 1= \\
\mathrm{L}: \mathrm{N}(05: 95)\end{array}$ & $\begin{array}{l}\mathrm{O} 1= \\
\mathrm{O}: \mathrm{N}(05: 95)\end{array}$ & $\begin{array}{l}\text { Pf1= } \\
\text { Pf:N(05:95) }\end{array}$ \\
\hline $\begin{array}{l}\text { S2= } \\
\text { S:N(10:90) }\end{array}$ & $\begin{array}{l}\mathrm{P} 2= \\
\mathrm{P}: \mathrm{N}(10: 90)\end{array}$ & $\begin{array}{l}\text { M2= } \\
\text { M:N(10:90) }\end{array}$ & $\begin{array}{l}\mathrm{L} 2= \\
\mathrm{L}: \mathrm{N}(10: 90)\end{array}$ & $\begin{array}{l}\mathrm{O} 2= \\
\mathrm{O}: \mathrm{N}(10: 90),\end{array}$ & $\begin{array}{l}\text { Pf } 2= \\
\text { Pf:N(10:90) }\end{array}$ \\
\hline $\begin{array}{l}\text { S3= } \\
S: N(15: 85\end{array}$ & $\begin{array}{l}\mathrm{P} 3= \\
\mathrm{P}: \mathrm{N}(15: 85)\end{array}$ & $\begin{array}{l}\text { M3= } \\
M: N(15: 85)\end{array}$ & $\begin{array}{l}\mathrm{L3}= \\
\mathrm{L}: \mathrm{N}(15: 85)\end{array}$ & $\begin{array}{l}\mathrm{O} 3= \\
\mathrm{O}: \mathrm{N}(15: 85)\end{array}$ & $\begin{array}{l}\text { Pf 3= } \\
\text { Pf:N(15:85) }\end{array}$ \\
\hline
\end{tabular}

Where,

$\mathrm{S}=1 \%$ aqueous solution of stearyl imidazolinium surfactants, $\mathrm{P}=1 \%$ aqueous solution of palmityl imidazolinium surfactants, $\mathrm{M}=1 \%$ aqueous solution of myristyl imidazolinium surfactants, $\mathrm{L}=1 \%$ aqueous solution of lauryl imidazolinium surfactants acid, $\mathrm{O}=1 \%$ aqueous solution of oleyl imidazolinium surfactants acid, $\mathrm{Pf}=1 \%$ aqueous solution of palm fatty acids based imidazolinium surfactants and $\mathrm{N}=1 \%$ aqueous solution of nonionic surfactant.

\subsubsection{Determination of foaming properties of cationic-nonionic compositions}

The foaming power of different compositions were determined by pouring each of them into $100 \mathrm{ml}$ stoppered measuring cylinder giving 30 constant vigorous strokes by hand (6) .The volume of the foam produced was read immediately and after $5 \mathrm{~min}$.

Softness, rewettability and detergency were evaluated on cotton fabric by different methods.

Fabric pretreatment: Test samples (fabrics) were cut to a size $4 \times 4$ in, for all experiments. All samples were boiled with distilled water for 30 minutes to remove water soluble impurities and unreacted finishing components. The fabric samples were dried in a drying oven at $105^{\circ} \mathrm{C}$ for 2 hours and stored in a dessicator.

Cationic surfactant treatment: $1 \%$ aqueous solutions of the cationic surfactant were prepared. A liquid to cloth ratio of 30:1 was selected. The dried fabric samples were weighed and appropriate volumes of treatment solution were put into $250 \mathrm{ml}$ beakers. The samples used to evaluate fabric properties were treated for $120 \mathrm{~min}$ at $35^{\circ} \mathrm{C}$. 
After treatment, fabric samples were removed from the beaker and padded between textile blotting paper less than $35 \mathrm{lb}$ of pressure. Samples were allowed to dry overnight on blotting paper and were stored in polyethylene bags for future evaluation.

\subsubsection{Determination of softening properties of cationic-nonionic compositions}

No good mechanical method has yet been devised for the quantitative measurement of softness. As a result, in the present study, a panel "feel" test (7) was used for softness evaluation of fabrics.

Procedure: A panel consisting of at least five people was asked to rate the softness of the test fabric. The panel members were allowed individually to feel the sets of test fabrics and rate their softness on a scale of 1 through 5 with 1 being the harshest and 5 being the softest.

\subsubsection{Determination of rewetting properties of cationic-nonionic compositions}

Rewetting properties are important because they give an indication of the absorbency characteristics of fabric after treatment with a softener. If too much fabric softener is used, it is possible to water proof the cloth. This attribute was measured by using a dye wick-up method (8).

Procedure: A $0.18 \%$ aqueous solution of Acid Blue (dye) was prepared and an aliquot placed in a graduated cylinder. One-to-two inch wide strips of cationic treated fabric were suspended into dye solution, using a glass rod sewn into the strip at the bottom for weight and a slitted card board at the top of the cylinder so that with the aid of paper clamps, the strip could be immersed to the depth of $3 \mathrm{~cm}$ in the dye solution. A stop watch was started as soon as the strip was immersed and centimeter height of dye solution was recorded at 5 minutes.

\subsubsection{Determination of detergency of cationic-nonionic compositions}

Detergency of surfactant compositions were determined as per BIS (9) methods with the help of tergot-ometer by using $4 \times 4 \mathrm{~cm}$ piece $100 \%$ cotton fabric and percentage removal of soil were calculated by formula:

Calculations

$\%$ SOIL REMOVAL $=$ A-B $/$ C-B $\times 100$

$\mathrm{A}=$ reflectance of soiled fabric after washing

$\mathrm{B}=$ reflectance of soiled fabric before washing

$\mathrm{C}=$ reflectance of white fabric before soiling

\subsection{Surface Active Properties Of Imidazolinium Surfactants}

\subsubsection{Surface tension and interfacial tension}

Surface tension and interfacial tension of $1 \%$ aqueous solutions of all six synthesized imidazolinium surfactants were studied at $28{ }^{\circ} \mathrm{C}$ and found that stearic acid based imidazolinium quat solution had $35.7 \mathrm{mN} / \mathrm{m}$ and $11.2 \mathrm{mN} / \mathrm{m}$ of surface tension and interfacial tension, respectively. Palmitic acid based imidazolinium quat solution showed comparatively lower surface tension and interfacial tension i.e. $31.4 \mathrm{mN} / \mathrm{m}$ and $9.8 \mathrm{mN} / \mathrm{m}$, respectively. Imidazolinium quats of myristic acid, lauric acid and oleic acid showed lower values of surface tension than palmitic acid based imidazolinium quats which were $29.4,24.1$ and $28.8 \mathrm{mN} / \mathrm{m}$, respectively and same pattern was found in case of interfacial tension of surfactant solutions. Palm fatty acids based imidazolinium quat solution showed $30.6 \mathrm{mN} / \mathrm{m}$ of surface tension and $9.1 \mathrm{mN} / \mathrm{m}$ of interfacial tension which were almost similar to the nonionic surfactant which was used as a reference. These results showed that in case of $\mathrm{C}_{12}-\mathrm{C}_{18}$ saturated fatty alkyl lipophilic groups, surface tension of $1 \%$ aqueous solution of imidazolinium salts decreased with the decrease in the chain length of lipophilic group. Oleic acid derivative showed lesser surface tension than its saturated homologue due to the presence of double bond while palm fatty acid based surfactant solution and nonionic surfactant showed almost similar surface tension.

\subsubsection{Critical micelle concentration}

CMC of all six surfactant solutions and nonionic surfactant solution was studied and was found in the range of $0.023 \mathrm{mmol} / \mathrm{l}$ to $0.025 \mathrm{mmol} / \mathrm{l}$. CMC of stearic acid based imidazolinium surfactants was found to be $0.022 \mathrm{mmol} / \mathrm{l}$. Imidazolinium surfactants based on palmitic acid, myristic acid, lauric acid and oleic acid gave cmc values of $0.024,0.026,0.027$ and $0.23 \mathrm{mmol} / \mathrm{l}$, respectively. Palm fatty acids based imidazolinium surfactants showed almost similar cmc value as that of nonionic surfactant which was found to be $0.25 \mathrm{mmol} / \mathrm{l}$. These results showed that $\mathrm{cmc}$ of surfactants increased with decrease in the chain length of hydrophobic group. Stearic acid based imidazolinium surfactant showed lowest value of $\mathrm{cmc}$ while lauric acid based imidazolinium surfactant showed highest $\mathrm{cmc}$ value. 


\subsubsection{Cationic content}

Cationic content of $1 \%$ aqueous solution of surfactants were determined to check their active content which were found to be 57.4, 59.8, 67.4, 70.2, 62.8 and 58.6\% for imidazolinium surfactants based on stearic acid, palmitic acid, myristic acid, lauric acid, oleic acid and palm fatty acids, respectively. Above observations showed that increase in the chain length of lipophilic group deccreased the cationic content of surfactants.

\subsubsection{Dispersing power}

Dispersing power of $1 \%$ aqueous solution of surfactants were measured in terms of volume of dispersed phase in $\mathrm{ml}$ and it was found that stearic acid based imidazolinium surfactant showed $10 \mathrm{ml}$ of dispersed phase. Imidazolinium surfactant solutions of palmitic acid, myristic acid, lauric acid, oleic acid showed dispersed phase $9 \mathrm{ml}, 7 \mathrm{ml}, 6 \mathrm{ml}$ and $5 \mathrm{ml}$, respectively. Surfactant based on palm fatty acids showed $7.5 \mathrm{ml}$ of dispersed phase while nonionic surfactant showed only $3 \mathrm{ml}$ of dispersed phase. These results depicted that cationic surfactant solutions showed better dispersing power in comparison to nonionic surfactant. Increase in the chain length of lipophilic group gradually increased the dispersing power of imidazolinium surfactants. Oleic acid based imidazolinium surfactant showed poorest dispersing power due to the presence of double bond.

\subsubsection{Emulsion stability}

Emulsion stability of surfactant solutions was measured in terms of time required for their separation from acetone layer. More the time required for separation, more was the emulsion stability of surfactants. For stearic acid based imidazolinium surfactants time required for separation was $3.45 \mathrm{~h}$ which gradually decreased to $3.20,3.00,2.5,3.25$ and $3.10 \mathrm{~h}$ for surfactants based on palmitic acid, myristic acid, lauric acid, oleic acid and palm fatty acids, respectively. Time required for separation of nonionic surfactant to acetone was $1.5 \mathrm{~h}$. These results revealed that nonionic surfactant solution shows poor emulsion stability as compared to cationic imidazolinium surfactants. In imidazolinium surfactants increase in the chain length of lipophilic group gradually increased the emulsion stability of surfactant solutions. Oleic acid based imidazolinium surfactant showed poor emulsion stability than its saturated homologue.

\subsection{Performance Properties Of Cationic- Nonionic Surfactant Compositions \\ 4.2.1. Foaming}

Foaming properties of cationic-nonionic surfactants solutions were measured in terms of foam volume in $\mathrm{ml}$ (Fig.2) and it was found that $100 \%$ nonionic surfactant showed $80 \mathrm{ml}$ foam volume. Introduction of cationic surfactant to nonionic surfactant decreased the foam volume of the compositions. For stearic acid based compositions, it was found $40 \mathrm{ml}$ for S1, 37 for S2, 32 for S3 and 30 for S4. Almost identical pattern was found for other cationic surfactant compositions. Solutions of $100 \%$ pure imidazolinium surfactants showed negligible foaming as compared to Triton X-100 solution. In laundry compositions of cationic-nonionic surfactants, increase in the concentration of cationic surfactant gradually decreased the foam height of surfactant compositions but increased the foam stability of compositions (Table 2).

In the case of imidazolinium salts of $\mathrm{C}_{12}-\mathrm{C}_{18}$ alkyl lipophilic chains, foam ability gradually decreased with increase in chain length of lipophilic group. On the contrary, oleic acid based imidazolinium surfactant showed best foaming ability with nonionic surfactant which may be due to the presence of double bond.

\subsubsection{Rewettability}

Rewettability of compositions was measured in terms of time (Fig. 3) required for rewetting of swatch by dye solution after their treatment with surfactants compositions. $100 \%$ pure imidazolinium surfactants showed very poor rewettability which was found to be $46,44,39,36,33$ and $42 \mathrm{~min}$ for stearic acid, palmitic acid, myristic acid, lauric acid, oleic acid and palm fatty acids based imidazolinium surfactants, respectively. Increasing the percentage of imidazolinium surfactant in laundry compositions from 5\% to $20 \%$ gradually decreased the rewettability of compositions. In laundry compositions of saturated imidazolinium salts, rewetting ability gradually decreased with increase in chain length of lipophilic group. On the other hand, compositions based on imidazolinium salts of oleic acid showed comparatively better rewetting power.

\subsubsection{Softening}

In cationic-nonionic surfactant compositions, increasing the percentage of cationic surfactant from 5\% to $20 \%$ gradually increased the softening ability of laundry compositions (Table 3). In saturated imidazolinium surfactants, softening property gradually increased with increase in chain length of lipophilic group. Imidazolinium surfactants showed better softening property as compared to nonionic surfactant (negligible softening). Laundry compositions based on imidazolinium salt of oleic acid showed comparatively poor softening to their saturated homologue. 


\subsubsection{Detergency}

Detergency of surfactant compositions were measured in terms of \% particulate soil removal (Fig.4). Triton X-100 (nonionic surfactant) showed good detergency i.e. 98.5\%. In laundry compositions, addition of cationic imidazolinium surfactants to nonionic surfactants gradually increased the detergency of surfactant compositions. Stearic acid based cationic- nonionic compositions (S1) showed $99.4 \%$ of soil removal. Increasing the percentage of cationic surfactant based on stearic acid from 5 to 10 percent increased the soil removal upto $100 \%$ which remained unaffected for 15 to 20 percent of stearic acid based cationic-nonionic surfactant compositions. Almost identical pattern of soil removal was observed for other cationic-nonionic compositions based on different fatty acids underquestion. This may be due to the increase in adsorption and emulsification behavior of surfactant composition after addition of cationic surfactant to nonionic surfactant. Fig. 4 showed that more the length of lipophilic chains of cationic surfactant, more the adsorption and emulsification hence more the detergency of compositions.

Table 2: $\quad$ Surface active properties of dialkyl quaternary imidazolinium salts of different fatty acids

\begin{tabular}{|lccccccc|}
\hline \multicolumn{1}{|c}{ Property } & $\mathrm{N}$ & $\mathrm{S}$ & $\mathrm{P}$ & $\mathrm{M}$ & $\mathrm{L}$ & $\mathrm{O}$ & Pf \\
\hline $\begin{array}{l}\text { Interfacial tension } \\
\text { (mN/m) }\end{array}$ & 8.9 & 11.2 & 9.8 & 7.9 & 8.7 & 8.5 & 9.1 \\
$\begin{array}{l}\text { Surface tension (mN/m) } \\
\text { at 28 } \mathbf{~ C}\end{array}$ & 30.2 & 35.7 & 31.4 & 24.1 & 29.2 & 28.8 & 30.6 \\
CMC mol/l & 0.025 & 0.022 & 0.024 & 0.026 & 0.027 & 0.023 & 0.025 \\
$\begin{array}{l}\text { Cationic content } \\
\text { \%) }\end{array}$ & - & 57.4 & 59.8 & 67.4 & 70.2 & 62.8 & 58.6 \\
$\begin{array}{l}\text { Dispersing power } \\
\text { (vol. in ml) }\end{array}$ & 3 & 10 & 9 & 7 & 6 & & \\
Emulsion stability (h) & 1.50 & 3.45 & 3.20 & 3.00 & 2.50 & 3.25 & 3.10 \\
\hline
\end{tabular}

$\mathrm{S}=1 \%$ aqueous solution of stearyl imidazolinium surfactants, $\mathrm{P}=1 \%$ aqueous solution of palmityl imidazolinium surfactants, $\mathrm{M}-1 \%$ aqueous solution of myristyl imidazolinium surfactants, $\mathrm{L}=1 \%$ aqueous solution of lauryl imidazolinium surfactants acid, $\mathrm{O}=1 \%$ aqueous solution of oleyl imidazolinium surfactants acid, $\mathrm{Pf}=1 \%$ aqueous solution of palm fatty acids based imidazolinium surfactants and $\mathrm{N}=1 \%$ aqueous solution of nonionic surfactant.

Table 3: $\quad$ Foam stability of various cationic-nonionic compositions

\begin{tabular}{|lcc|}
\hline S. No. & Surfactant type & $\begin{array}{c}\text { Foam stability* } \\
(\%)\end{array}$ \\
\hline 1 & N & 65 \\
2 & S1 & 87 \\
3 & S2 & 89 \\
4 & S3 & 90 \\
5 & S4 & 97 \\
6 & P1 & 88 \\
7 & P2 & 91 \\
8 & P3 & 95 \\
9 & P4 & 98 \\
10 & M1 & 85 \\
11 & M2 & 87 \\
12 & M3 & 90 \\
13 & M4 & 93 \\
14 & L1 & 82 \\
15 & L2 & 86 \\
16 & L3 & 89 \\
17 & L4 & 91 \\
18 & O1 & 74 \\
\hline
\end{tabular}




\begin{tabular}{|lll|}
\hline 19 & O2 & 81 \\
20 & O3 & 86 \\
21 & O4 & 88 \\
22 & Pf1 & 88 \\
23 & Pf2 & 90 \\
24 & Pf3 & 92 \\
25 & Pf4 & 98 \\
\hline
\end{tabular}

*average of two determinations

Table 4: $\quad$ Softening properties of various cationic-nonionic compositions

\begin{tabular}{|c|c|c|}
\hline S. No. & Surfactant type & Rating \\
\hline 1 & $\mathrm{~N}$ & Nil \\
\hline 2 & S & ++++++++++ \\
\hline 3 & S1 & ++++++ \\
\hline 4 & $\mathrm{~S} 2$ & +++++++ \\
\hline 5 & S3 & ++++++++ \\
\hline 6 & S4 & ++++++++++ \\
\hline 7 & $\mathrm{P}$ & ++++++++++ \\
\hline 8 & P1 & +++++ \\
\hline 9 & $\mathrm{P} 2$ & ++++++ \\
\hline 10 & P3 & +++++++ \\
\hline 11 & P4 & ++++++++ \\
\hline 12 & M & +++++++ \\
\hline 13 & M1 & +++ \\
\hline $\begin{array}{l}14 \\
15\end{array}$ & $\begin{array}{l}\text { M2 } \\
\text { M3 }\end{array}$ & $\begin{array}{c}++++ \\
+++++\end{array}$ \\
\hline 16 & M4 & ++++++ \\
\hline 17 & $\mathrm{~L}$ & ++++++ \\
\hline 18 & $\mathrm{~L} 1$ & ++ \\
\hline 19 & L2 & +++ \\
\hline 20 & L3 & ++++ \\
\hline 21 & L4 & +++++ \\
\hline 22 & $\mathrm{O}$ & +++++ \\
\hline 23 & $\mathrm{O} 1$ & + \\
\hline 24 & $\mathrm{O} 2$ & ++ \\
\hline 25 & $\mathrm{O} 3$ & +++ \\
\hline 26 & $\mathrm{O} 4$ & ++++ \\
\hline 27 & $\mathrm{Pf}$ & +++++++++ \\
\hline 28 & Pf1 & ++++ \\
\hline 29 & Pf2 & +++++ \\
\hline 30 & Pf3 & ++++++ \\
\hline 31 & Pf4 & +++++++ \\
\hline
\end{tabular}




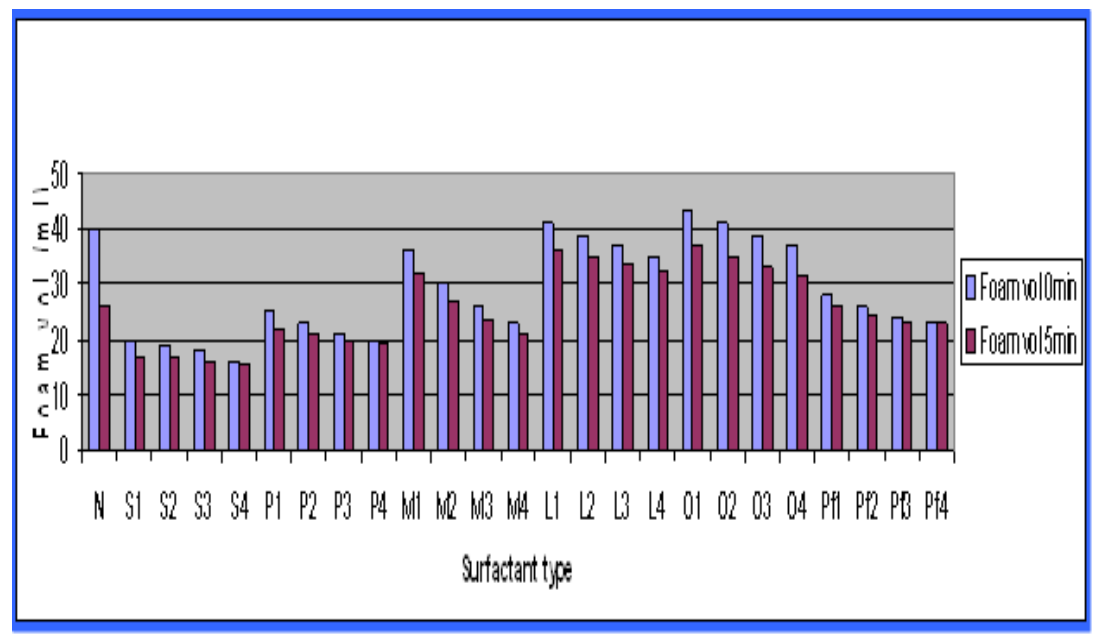

Fig.2: Foaming properties of various cationic-nonionic compositions.

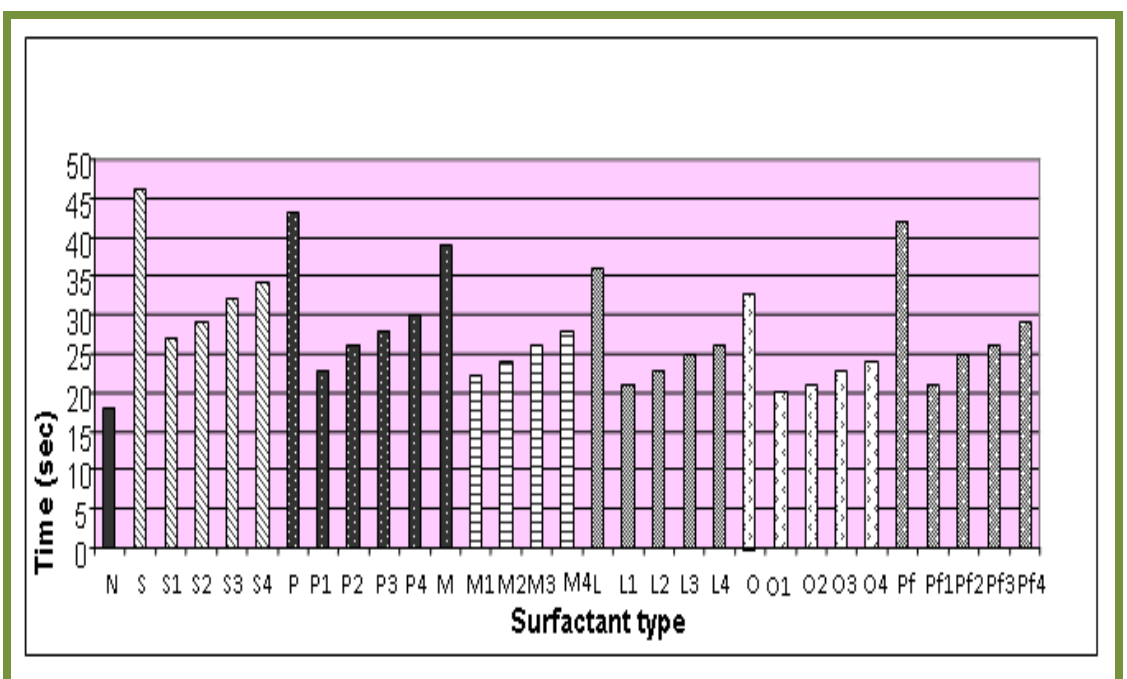

Fig.3: Rewettability of various cationic-nonionic compositions

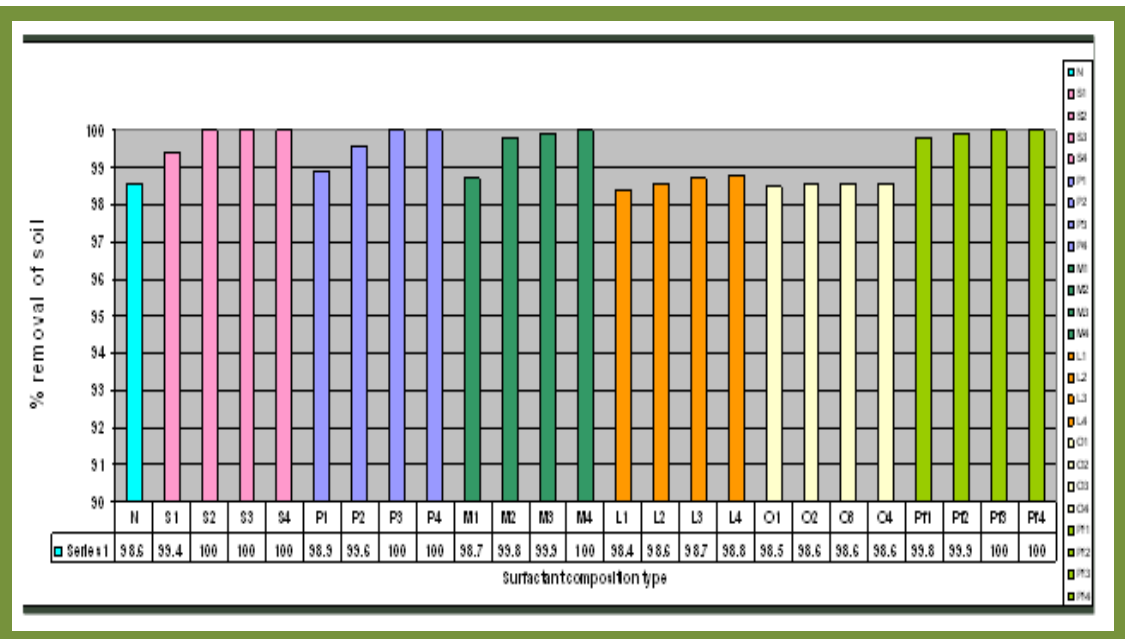

Fig.4: Detergency of various cationic-nonionic compositions 


\section{References}

[1]. P. R. Gerardo , D. P. Jesus, M. Cruz, L. G. Zepeda, "Synthesis of long chain 2-alkyl-1-(2-hydroxy ethyl)-2-imidazolines under microwave in solvent free conditions", Synlett, 12: 1847-1849(2003).

[2]. Laundry detergent, Wikipedia, the free encyclopedia.

[3]. K. Hoffmann, The Chemistry of Heterocyclic Compounds, Interscience Publishers, Inc., New York, London, pp.213-26(1953).

[4]. K. Hamirin, S. Nakasato and M. A. Rahman, Imidazoline Amphoteric Surfactants from Palm and Palm Kernel Fatty Acids, ELAEIS, 3 (1), 296 (1991).

[5]. H. Takeshi, Bull. Chem. Soc. Japan, 43: 2236-2240 (1970).

[6]. L. Perez, A. Pinazo, M.J. Rosen, and M.R. Infante, , Langmuir, Vol. 14, 2307, (1998).

[7]. M. E. Ginn, T. A. Schenach and E. Jungermann, Ibid, 42, 1084 (1965).

[8]. R. R. Egan, J. Amer. Oil Chem. Soc., 55, 118 (1978).

[9]. Indian Standard Methods for performance Tests For Surface Active Agents, Part IV Relative Detergency (1976). 